\title{
A prospective randomized trial examining health care utilization in individuals using multiple smartphone-enabled biosensors
}

Cinnamon S Bloss, Nathan E Wineinger, Melissa Peters, Debra L Boeldt, Lauren Ariniello, Ju Young Kim, Judith Sheard, Ravi Komatireddy, Paddy Barrett, Eric J Topol

Background. Mobile health and digital medicine technologies are becoming increasingly used by individuals with common, chronic diseases to monitor their health. Numerous devices, sensors, and apps are available to patients and consumers - some of which have been shown to lead to improved health management and health outcomes. However, no randomized controlled trials have been conducted which examine health care costs, and most have failed to provide study participants with a truly comprehensive monitoring system. Methods. We conducted a prospective randomized controlled trial of adults who had submitted a 2012 health insurance claim associated with hypertension, diabetes, and/or cardiac arrhythmia. The intervention involved receipt of one or more mobile devices that corresponded to their condition(s) and an iPhone with linked tracking applications for a period of 6 months; the control group received a standard disease management program. Moreover, intervention study participants received access to an online health management system which provided participants detailed device tracking information over the course of the study. This was a monitoring system designed by leveraging collaborations with device manufacturers, a connected health leader, health care provider, and employee wellness program - making it both unique and inclusive. We hypothesized that health resource utilization with respect to health insurance claims may be influenced by the monitoring intervention. We also examined health-self management. Results $\boldsymbol{\&}$ Conclusions. There was little evidence of differences in health care costs or utilization as a result of the intervention. Furthermore, we found evidence that the control and intervention groups were equivalent with respect to most health care utilization outcomes. This result suggests there are not large short-term increases or decreases in health care costs or utilization associated with monitoring chronic health conditions using mobile health or digital medicine technologies. Among secondary outcomes there was some evidence of improvement in health self-management which was characterized by a decrease in the propensity to view health status as due to chance factors in the intervention group. 


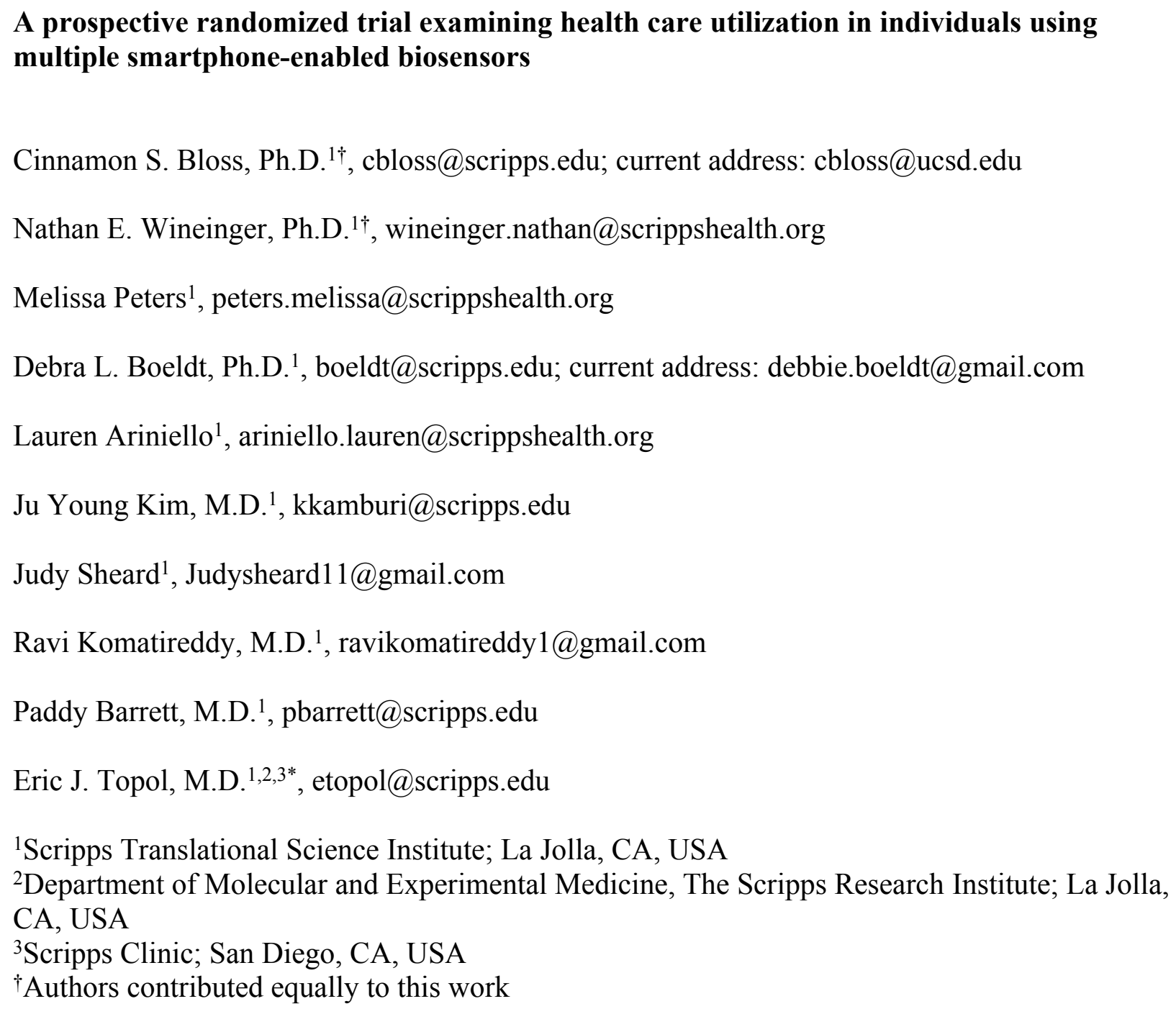

Short title: Prospective randomized digital medicine trial

Keywords: digital medicine, mobile health, health monitoring, hypertension, diabetes, arrhythmia, health insurance claims

*Address for Correspondence: $\quad$ Eric J. Topol, M.D.

Scripps Translational Science Institute

3344 N. Torrey Pines Court, Suite 300

La Jolla, California 92037

Phone: (858) 554-5708

Fax: (858) 546-9272

etopol@scripps.edu

Disclosure of Funding: This research is funded in part by a NIH/NCATS flagship Clinical and

46 Translational Science Award Grant (1UL1 TR001114), Qualcomm Foundation Scripps Health 
47 Digital Medicine Research Grant, and Scripps Health's Division of Innovation and Human 48 Capital and Division of Scripps Genomic Medicine. Support for the study is also provided by 49 HealthComp Third Party Administrator, Sanofi, AliveCor, and Accenture.

50

51 Trial Registration: clinicaltrials.gov Identifier NCT01975428

52

53 CONSORT flowchart is found in Supplemental Figure 7 (i.e., Figure S7). 


\section{ABSTRACT}

55 Background. Mobile health and digital medicine technologies are becoming increasingly used by individuals with common, chronic diseases to monitor their health. Numerous devices, sensors, and apps are available to patients and consumers - some of which have been shown to lead to improved health management and health outcomes. However, no randomized controlled trials have been conducted which examine health care costs, and most have failed to provide study participants with a truly comprehensive monitoring system.

Methods. We conducted a prospective randomized controlled trial of adults who had submitted a 2012 health insurance claim associated with hypertension, diabetes, and/or cardiac arrhythmia.

The intervention involved receipt of one or more mobile devices that corresponded to their condition(s) (hypertension: Withings Blood Pressure Monitor; diabetes: Sanofi iBGStar Blood Glucose Meter; arrhythmia: Alivecor Mobile ECG) and an iPhone with linked tracking applications for a period of 6 months; the control group received a standard disease management program. Moreover, intervention study participants received access to an online health management system which provided participants detailed device tracking information over the course of the study. This was a monitoring system designed by leveraging collaborations with device manufacturers, a connected health leader, health care provider, and employee wellness

71 program - making it both unique and inclusive. We hypothesized that health resource utilization with respect to health insurance claims may be influenced by the monitoring intervention. We 73 also examined health-self management.

74 Results \& Conclusions. There was little evidence of differences in health care costs or utilization as a result of the intervention. Furthermore, we found evidence that the control and intervention groups were equivalent with respect to most health care utilization outcomes. This 
77 result suggests there are not large short-term increases or decreases in health care costs or

78 utilization associated with monitoring chronic health conditions using mobile health or digital

79 medicine technologies. Among secondary outcomes there was some evidence of improvement in

80 health self-management which was characterized by a decrease in the propensity to view health

81 status as due to chance factors in the intervention group. 


\section{INTRODUCTION}

83 Hypertension, diabetes mellitus, and cardiac arrhythmias are chronic diseases with a significant

84 health burden. The high prevalence and well-characterized complications of these conditions

85 result in negative impacts to quality of life, morbidity, and mortality. Vast medical, scientific,

86 and engineering resources have been devoted in efforts to find ways to improve diagnosis,

87 treatment, management, and prevention, including advances in diagnostic technology (Willits et

88 al., 2014; Zhang et al., 2014; "National Institute for Health and Care Excellence,” 2015),

89 dissemination of identifiable risk factors (Wilson et al., 1998; Chobanian et al., 2003; American

90 Diabetes Association, 2014), and development of pharmaceuticals (The ALLHAT Officers and

91 Coordinators for the ALLHAT Collaborative Research Group, 2000; The ALLHAT Officers,

92 2002; Uzu et al., 2005; Ernst et al., 2006). Nevertheless, the continued maintenance of these

93 efforts and the costs associated with direct patient care of individuals with these conditions

94 remains a sizable fraction of health care costs (Kim et al., 2011; Davis, 2013; Yang et al., 2013).

95

96 Despite these efforts, the management of these conditions remains challenging (Hansen et al.,

97 2005). Patient engagement, medication adherence, and adherence to treatment strategies is

98 variable and often suspect (Guyatt et al., 1986; Hansen et al., 2005). Furthermore, poor

99 communication between patients and their health care providers can accentuate these issues. The

100 end result is often a major clinical decompensation event that could have been largely

101 preventable. In addition to these clinical consequences, these challenges also result in economic

102 consequences such as high utilization of inpatient resources and emergency departments, as well

103 as readmissions (Sander \& Giles, 2011). One would surmise that better, more informed 
104 management of disease would lead to better long-term health outcomes and thereby lower health

105 care resource utilization.

106

107 Understanding both this problem of day-to-day poor health care management and ubiquity of

108 smartphones and other mobile computing platforms in our daily lives, numerous device

109 manufacturers have developed biomedical sensors designed for patient consumers which

110 measure meaningful physiologic metrics ("National Institute for Health and Care Excellence,"

111 2015). These sensors often utilize a smartphone to display information, while some can employ

112 the internet network connectivity of the smartphone to transmit data to remote servers for

113 additional display, storage, or analytics. Individuals appropriately using such devices can

114 monitor their condition in their own real world setting - potentially making management of

115 disease more personalized and more engaging. This data may provide individuals with early

116 recognition of disease symptoms and consequences of behaviors, which can allow patients and

117 providers to make proactive health care decisions, thereby leading to improved health outcomes

118 and potentially reduce health care utilization. However, there is potential that such monitoring

119 may lead to a short-term increase in health care resource utilization even if appropriate, or over-

120 utilization while patients are learning to recognize which readings constitute normal variation

121 and which readings indicate a health issue requiring medical attention.

122

123 In large part, the jury is still out if chronic disease monitoring using mobile health and digital

124 medicine technology will, on its own, improve health outcomes (Steinhubl, Muse \& Topol, 2013,

125 2015). Many previous studies have shown improvements, but many others have shown none

126 (Free et al., 2013a,b; Hamine et al., 2015; Karhula et al., 2015). Whether or not a patient has 
127 improved health as a result such monitoring likely depends on the behavior of the individual and

128 the technology itself. Motivated individuals using an informative device which captures

129 actionable data are likely to see improvements, while unmotivated individuals using devices

130 which capture meaningless or nonactionable information will see no benefit. What has yet to be

131 fully explored is how such monitoring will impact health care utilization if digital medicine

132 technology becomes embraced by the medical establishment in the face of an increasingly

133 informed, technology-embracing patient population (Boeldt et al., 2015).

135 In light of this, we conducted a prospective, randomized-controlled trial designed to assess the

136 impact of mobile health monitoring on short-term health care utilization in individuals with

137 hypertension, diabetes, or arrhythmia. The intervention consisted of a comprehensive, integrated

138 monitoring system that included wireless medical devices designed to be used with a

139 smartphone, a smartphone with appropriate monitoring applications, a web-based care

140 management portal and iOS-based mobile application where patients could access their data, and

141 a staff of nurses and technicians available for assistance. Given the potential for both short-term

142 and long-term impacts on health care utilization, we hypothesized that the intervention may

143 impact health care costs compared to standard disease management practices. We also examined

144 how this intervention influenced health self-management. We conducted this mobile health

145 management strategy on 160 employees and dependents from a large health care system.

147 MATERIALS \& METHODS

148 Study design 
149 The research design was a prospective, simple randomized controlled, two-group, pre-post

150 intervention trial. Of 21,691 individuals insured by Scripps Health (employees and dependents)

151 and who submitted at least one claim in 2012, 3,998 individuals age 18 or over who had billed a

152 claim with a current procedural terminology (CPT) code related to hypertension, insulin-

153 dependent or non-insulin dependent diabetes, and/or with arrhythmia were identified and, if

154 eligible, offered study participation. No stratification with respect to condition was employed.

155 Additional inclusion and exclusion criteria are included in Supplemental Methods. The study

156 period was six months and the trial took place between July 2013 and December 2014. The study

157 was approved by the Scripps Institutional Review Board (approval \# IRB-12-6019).

159 Study recruitment

160 The 3,998 eligible individuals were ranked according to the amount of their 2012 health

161 insurance claims billed for the three study conditions. Recruitment proceeded in blocks starting

162 with individuals in the highest $25 \%$ in terms of dollar amount, then the highest $50 \%$ and so on.

163 For each block of individuals a letter was sent describing the trial and disease management

164 program. Within two to four weeks the letter was followed up with a telephone call made by a

165 HealthComp nursing staff member in which the study was explained. HealthComp is the third

166 party administrator for Scripps Health. A maximum of three calls were attempted before a

167 prospective participant was considered not reachable. For those prospective participants who

168 expressed interest, a link to the online informed consent was sent via email. Prospective

169 participants were asked to read and sign the online consent. Once consented, participants were

170 directed to complete an online baseline survey. Afterwards, participants were randomized to

171 control or intervention and brought in for an enrollment visit with an unblinded research 
172 coordinator. The participants were blind to their assigned group prior to enrollment. It was

173 explained to all participants that their employer would not have access to any of their medical

174 information used for the study.

175

176 Study enrollment

177 At the enrollment study visit, individuals assigned to the intervention arm were provided with a 178 study iPhone 4 or $4 \mathrm{~s}$ (even if they owned one) and one or more mobile devices that corresponded 179 to their condition(s): a Withings Blood Pressure Monitor (hypertension), Sanofi iGBStar Blood 180 Glucose Meter (diabetes), or AliveCor Mobile ECG (arrhythmia). As part of the intervention, 181 participants were also supplied with an online account to HealthyCircles ${ }^{\mathrm{TM}}$. HealthyCircles is a

182 Qualcomm Life health care coordination and management platform with an integrated suite of 183 management and consumer portals that can deliver chronic disease education and connect users

184 to their families, caregivers, and health care professionals. As part of the study, HealthComp nursing staff had access to the HealthyCircles care management dashboard which displayed the participant's device monitoring results and trends over time. Device readings collected by the participant were wirelessly uploaded to the patient's HealthyCircles account and made available to the HealthComp nurses as well as the patient via the study phone or a computer. Example displays are included in Figure S1-S3. Also included in the management platform were reminders for monitoring, information about the participant's disease condition, and general

191 health behavior recommendations. Participants randomized to the intervention group were

192 trained on how to use their phone, the HealthyCircles mobile applications and portal, and their 193 medical device(s). All study participants, including participants randomized to the control arm,

194 were enrolled in the HealthComp disease management program, which involved outreach by 
195 HealthComp nursing staff for purposes of relaying medical education and wellness information

196 with regard to disease prevention and chronic disease management. In addition, systolic and

197 diastolic blood pressure readings were recorded at baseline and follow-up on individuals with

198 hypertension, whereas blood glucose and hemoglobin A1c levels were recorded on diabetics.

199 Participants were also provided with a contact email and phone number they could use to reach a 200 study staff member for technical and other study support.

201

202 Participants in the monitoring group were asked to take readings as follows: hypertension: twice

203 per day, three days per week, first one in the morning; insulin-dependent diabetes: three times

204 per day, once before each meal, and once before bed every day; non-insulin dependent diabetes:

205 once per day before meal, three times per week; and arrhythmia: when symptomatic (Table S4).

206 If their monitoring fell below the level defined in the "Poor Compliance" range, the HealthComp

207 nurse would send a secure email through the HealthyCircles message center reminding them of

208 the monitoring schedule. They were also asked some compliance-related questions and provided

209 with strategies for getting back on schedule with the program. If the participant's physician

210 recommended a monitoring schedule that was more frequent than that required for the study,

211 participants were encouraged to follow their physician's instructions. Also, if participants

212 experienced other symptoms, for example hypertension: visual changes, "bounding” pulse, chest

213 discomfort, nausea; diabetes: fatigue, visual changes, pre-syncopal symptoms, dyspnea, nausea,

214 vomiting; arrhythmia: chest discomfort, palpitations, rapid heart rate, feeling of "skipped beats,"

215 dyspnea, nausea, pre-syncopal symptoms they were recommended to take additional

216 measurements. Individuals were instructed on how to navigate the online disease management

217 program at http://connect.healthcomp.com. 


\section{Study procedures}

220 Study participants were asked to attend both an enrollment study visit (baseline) and end-of-

221 study visit (follow-up) six months later, and to complete both a baseline and follow-up survey on

222 SurveyMonkey. At the mid-point of the study (i.e. three months), each participant also received

223 an email seeking any feedback about their experience thus far, or asking if they were having any

224 problems or had any questions.

\section{Outcome measures}

227 For both the treatment and control groups, outcomes were assessed using claims data during the

228 enrollment and termination visits. Primary outcomes were health care resource utilization as

229 measured by health insurance claims and visits to the hospital during the study period (details

230 below). Secondary outcomes were health self-management as indicated by validated measures of

231 health locus of control (Wallston, Stein \& Smith, 1994), health self-efficacy (Lorig et al., 1989),

232 and patient activation (Hibbard et al., 2005).

233

\section{Health insurance claims}

235 All health insurance claims from January 1, 2013 through December 31, 2014 were collected on 236 each study participant. For each individual, the total claims, condition-specific claims,

237 pharmaceutical claims amounts (all in dollars) were calculated. Condition-specific claims were 238 the total amount in claims related to one of the three study conditions monitored. For example,

239 the amount of hypertension claims was the amount in claims in dollars with an ICD-9 code for

240 hypertension. Totals were calculated for a period of 6 months prior to study enrollment (baseline 
241 claims; Table S5) and 6 months during enrollment (enrollment claims; Table S6). Claims were

242 further partitioned into four categories: office visits, emergency room visits, inpatient stays, and

243 all visits (all in number of occurrences). Differences in enrollment claims were then compared to

244 baseline claims between the control and monitoring groups, as well as between groups with

245 specific conditions. Data are available in Supplemental Data.

247 Health self-management

248 Information on health self-management was collected through the baseline and follow-up survey.

249 The outcomes of interest were: 1) the four subscales of health locus of control (Internal, Chance,

250 Doctor, Others) as assessed by the Form C of the Multidimensional Health Locus of Control

251 (MHLC) 18-item scale (Wallston, Stein \& Smith, 1994); 2) health self-efficacy as assessed by

252 the Stanford Patient Education Research Center (PERC) 6-item scale (Lorig et al., 1989); and 3)

253 patient activation using the Patient Activation Measure 13-item measure (Hibbard et al., 2005).

254 Health locus of control measures an individual's belief that his or her health is dependent on

255 factors within his or her control; health self-efficacy measures an individual's confidence

256 managing his or her health; and patient activation measures an individual's skills and knowledge

257 in managing his or her own health.

258

259 Device usage

260 Device usage statistics were recorded for each study participant in the monitoring group.

261 Whenever a study participant used a device, the time, date, and user information of that

262 particular reading was sent to a database managed by Qualcomm and available to participants

263 through Healthy Circles. In the case of the Withings Blood Pressure Monitor and IBG Star, the 
264 reading measurements (i.e. blood pressure and blood glucose levels) were also recorded; while

265 PDFs corresponding to the AliveCor arrhythmia assessments were saved. An issue was

266 encountered where a subset of Withings measures could not be accurately determined. These

267 measures were omitted. There were 21 study participants affected in varying severity, 10 of

268 which had this issue present in all data and 8 others had this issue present in at least $16 \%$ of the

269 data (the other two $6 \%$ and $0.5 \%$ ). As accurate device usage information on these 18 individuals

270 could not be determined, it was treated as missing. For all other study participants, the total

271 number of readings taken on each device was recorded.

272

273 Statistical analyses \& sample size justification

274 Between group differences were compared using a paired two sample t-test or Mann-Whitney

275 test in cases of small sample sizes and skewed outcomes (e.g. health insurance claims). By using

276 this paired approach we better model the change in outcomes of interest induced by the

277 intervention and reduce the influence of baseline confounders in the association statistics.

278 Equivalence testing was performed using the two one-sided test for equivalence using a

279 magnitude of region of similarity equal to half a standard deviation for each outcome The study

280 was designed to be powered (a priori) to detect a one office visit difference between the control

281 and monitoring arm (assuming a standard deviation of two office visits).

282

283 RESULTS

284 Participant demographics and information

285 Study participant demographics are presented in Table 1. Participants in the control and

286 monitoring groups were roughly equivalent with respect to common demographics and disease, 
287 which is consistent with the randomization process. A total of 89 had only hypertension, 9 non288 insulin dependent diabetes, 6 arrhythmia, 5 insulin-dependent diabetes, and 51 with more than 289 one of these conditions. The study enrollment flow chart is presented in Figure S7. Of the 160 290 individuals enrolled in the study, 130 completed both the baseline and follow-up assessments 291 ( $\mathrm{n}=65$ control, $\mathrm{n}=65$ monitoring; $\mathrm{p}=0.14)$. Using Google Analytics we observed a total of 3,670 292 sessions (after quality control filtering) to the HealthyCircles online disease management 293 program over the course of the study (Figure S8), with 7.17 page visits per session, and average 294 session duration of 11 minutes and 18 seconds. Google Analytics does not provide easily 295 accessible individual user website traffic data. We assessed weekly compliance of the 296 intervention in the monitoring group based on device usage (e.g., an individual with hypertension 297 would be compliant in a given week if they used the device at least six times that week). We 298 observed compliance rates were largely uniform (mean $=50 \%$ ), with $66 \%$ of individuals deemed 299 compliant at least one-third of the weeks. 
300 Table 1. Study participant demographics. Values are in counts, proportions in parentheses

301 (proportions) unless otherwise noted.

\begin{tabular}{|c|c|c|c|}
\hline & Monitoring & Control & $\mathrm{p}$-value \\
\hline N (\# completed) & $75(65)$ & $85(65)$ & 0.47 \\
\hline Hypertension & $67(89)$ & $71(84)$ & 0.29 \\
\hline NIDDM & $10(13)$ & $17(20)$ & 0.26 \\
\hline IDDM & $10(13)$ & $10(12)$ & 0.76 \\
\hline Arrhythmia & $10(13)$ & $19(22)$ & 0.14 \\
\hline Comorbidity & $21(28)$ & $30(35)$ & 0.41 \\
\hline Gender (\% Female) & $50(67)$ & $62(73)$ & 0.24 \\
\hline Age, Mean (SD) & $56(9.0)$ & $55(9.8)$ & 0.45 \\
\hline Ethnicity, Caucasian & $57(76)$ & $62(73)$ & 0.39 \\
\hline Education & & & 0.25 \\
\hline High School or Less & $10(13)$ & $19(22)$ & \\
\hline College & $32(43)$ & $37(44)$ & \\
\hline More than College & $33(44)$ & $29(34)$ & \\
\hline Family Size & & & 0.87 \\
\hline Single & $12(16)$ & $13(15)$ & \\
\hline Two & $27(36)$ & $34(40)$ & \\
\hline Three or More & $36(48)$ & $38(45)$ & \\
\hline Income & & & 0.09 \\
\hline$<\$ 50,000$ & $10(13)$ & $11(13)$ & \\
\hline$\$ 50 \mathrm{k}-\$ 149 \mathrm{k}$ & $47(63)$ & $58(68)$ & \\
\hline$>\$ 149 \mathrm{k}$ & $18(24)$ & $16(19)$ & \\
\hline Current Non-Smoker & $45(60)$ & $64(75)$ & 0.04 \\
\hline $\begin{array}{l}\text { Alcohol Use } \\
<1 / \text { week }\end{array}$ & $54(72)$ & $65(77)$ & 0.31 \\
\hline Active Exerciser & $37(49)$ & $37(44)$ & 0.46 \\
\hline Smartphone owned & & & 0.76 \\
\hline Did not own & $11(17)$ & $10(15)$ & \\
\hline Owned non-iPhone & $20(31)$ & $24(37)$ & \\
\hline Owned iPhone & $34(52)$ & $31(48)$ & \\
\hline
\end{tabular}

302 


\section{Health insurance claims}

304 Health insurance claims during the period of 6 months prior to study enrollment did not differ

305 between control and monitoring groups (Table S5). The average total amount of health insurance

306 claims during this period was $\$ 5,712(\mathrm{sd}=\$ 19,234$; median=\$976), and we observed no

307 difference in claims between individuals with different disease conditions $(p=0.99)$. The average

308 number of office visits was $4.1(\mathrm{sd}=4.2$; median=3); the average number of emergency room

309 visits was $0.10(\mathrm{sd}=0.45$; median $=0)$; and the average number of inpatient stays was 0.53

$310(\mathrm{sd}=3.10 ;$ median $=0)$. None of these claim categories differed statistically between conditions.

311

312 We did not observe any differences in health insurance claims between control and monitoring

313 groups during the 6 months of study enrollment (Table S6). This trend also persisted when we

314 accounted for baseline claims (Table 2). The average total amount of health insurance claims in

315 the monitoring group was $\$ 6,026$ while the average amount in the control group was $\$ 5,596$

$316(p=0.62)$. We note these averages are consistent with average total amount in health insurance

317 claims across the entire sampling frame (mean $=\$ 5,305)$, indicating that health insurance claims

318 in the monitoring group were not grossly different from the average patient (i.e., individuals not

319 enrolled in the study). We also did not observe any differences between the groups with respect

320 to office visits $(p=0.46)$, inpatient stays $(p=0.82)$, emergency room visits $(p=0.06)$, or pharmacy

321 claims $(\mathrm{p}=0.60)$. The total health insurance claims amount during enrollment also did not differ

322 by condition ( $\mathrm{p}=0.50)$, and we similarly observed no differences in claims specific to each

323 condition or multiple conditions (Table S6). 
325 Table 2. Health care utilization outcomes. Top: mean (standard deviation); bottom: median (IQR). $\mathrm{P}_{\text {Diff }}=\mathrm{p}$-value testing difference

326 between control and monitoring group; $\mathrm{P}_{\text {Equiv }}=\mathrm{p}$-value testing equivalence between groups; $*=$ Median and IQR all zero.

\begin{tabular}{|c|c|c|c|c|c|c|c|c|}
\hline & \multicolumn{2}{|l|}{ Baseline } & \multicolumn{2}{|l|}{ Follow-up } & \multicolumn{2}{|c|}{ Mean Difference } & \multirow[b]{2}{*}{$\mathbf{P}_{\text {Diff }}$} & \multirow[b]{2}{*}{$\mathbf{P}_{\text {Equiv }}$} \\
\hline & $\begin{array}{l}\text { Control } \\
N=85\end{array}$ & $\begin{array}{l}\text { Monitoring } \\
N=75\end{array}$ & $\begin{array}{l}\text { Control } \\
N=65\end{array}$ & $\begin{array}{l}\text { Monitoring } \\
N=65\end{array}$ & $\begin{array}{l}\text { Control } \\
N=65\end{array}$ & $\begin{array}{l}\text { Monitoring } \\
N=65\end{array}$ & & \\
\hline Total Claims (\$) & $\begin{array}{l}4,265 \\
(10,190) \\
961(3,166)\end{array}$ & $\begin{array}{l}7,159 \\
(25,251) \\
990(2,340)\end{array}$ & $\begin{array}{l}5,596 \\
(22,187) \\
807(2,734) \\
\end{array}$ & $\begin{array}{l}6,026 \\
(21,426) \\
845(2,273) \\
\end{array}$ & $\begin{array}{l}1,331 \\
(21,042) \\
0(2,372) \\
\end{array}$ & $\begin{array}{l}-1,133 \\
(31,465) \\
0(1,780) \\
\end{array}$ & 0.62 & 0.027 \\
\hline Condition Claims (\$) & $\begin{array}{l}1,512(6,868) \\
163(375)\end{array}$ & $\begin{array}{l}2,434 \\
(14,296) \\
117(387) \\
\end{array}$ & $\begin{array}{l}6,165 \\
(37,153) \\
111(379) \\
\end{array}$ & $\begin{array}{l}630(21,43) \\
179(516)\end{array}$ & $\begin{array}{l}4,653 \\
(35,795) \\
0(208) \\
\end{array}$ & $\begin{array}{l}-1,805 \\
(14,406) \\
0(283)\end{array}$ & 0.50 & 0.105 \\
\hline Pharmacy Claims (\$) & $\begin{array}{l}1,519(2,687) \\
325(1,590)\end{array}$ & $\begin{array}{l}1,859(5,315) \\
345(1,164) \\
\end{array}$ & $\begin{array}{l}1,667(2,780) \\
611(1,603) \\
\end{array}$ & $\begin{array}{l}2,188(6,340) \\
340(1,458)\end{array}$ & $\begin{array}{l}147(1,057) \\
11(531)\end{array}$ & $\begin{array}{l}329(1,860) \\
0(321)\end{array}$ & 0.60 & 0.037 \\
\hline Total Visits (\#) & $\begin{array}{l}4.49(5.01) \\
3(6)\end{array}$ & $\begin{array}{l}4.92(6.51) \\
3(4)\end{array}$ & $\begin{array}{l}4.17(4.21) \\
2(7)\end{array}$ & $\begin{array}{l}4.77(5.35) \\
3(5)\end{array}$ & $\begin{array}{l}-0.32(3.75) \\
0(2)\end{array}$ & $\begin{array}{l}-0.15(6.35) \\
0(3)\end{array}$ & 0.57 & 0.014 \\
\hline Office Visits (\#) & $\begin{array}{l}4.11(4.41) \\
3(5)\end{array}$ & $\begin{array}{l}4.05(4.09) \\
3(4)\end{array}$ & $\begin{array}{l}3.95(3.92) \\
2(5)\end{array}$ & $\begin{array}{l}4.32(4.48) \\
3(4)\end{array}$ & $\begin{array}{l}-0.15(3.30) \\
0(2)\end{array}$ & $\begin{array}{l}0.28(3.60) \\
0(2)\end{array}$ & 0.46 & 0.038 \\
\hline ER Visits $(\#)^{*}$ & $0.17(0.60)$ & $0.03(0.17)$ & $0.05(0.37)$ & $0.06(0.30)$ & $-0.12(0.72)$ & $0.03(0.35)$ & 0.06 & 0.137 \\
\hline Inpatient Stays (\#)* & $0.22(0.94)$ & $0.85(4.27)$ & $0.17(0.89)$ & $0.38(1.88)$ & $-0.05(1.16)$ & $-0.46(4.30)$ & 0.82 & 0.042 \\
\hline
\end{tabular}


328 Alternatively, we examined the differences in health care utilization using an equivalence testing

329 approach. Using a magnitude of region of similarity equal to half a standard deviation for each

330 outcome, in general we discovered that health care utilization was roughly equivalent between

331 groups (Table 2). We discovered that monitoring and control groups were roughly equal with

332 respect to total health insurance claims dollars $(\mathrm{p}=0.027)$, pharmacy claims $(\mathrm{p}=0.037)$, office

333 visits $(\mathrm{p}=0.038)$, inpatient stays $(\mathrm{p}=0.042)$, and total hospital visits $(\mathrm{p}=0.014)$. This suggests that

334 there is unlikely to be substantial short-term changes in health care utilization as a result of the

335 monitoring intervention.

336

337 We also examined health insurance utilization in a subset of the monitoring group who we were

338 able to assess as being compliant with the study protocol in at least one-third of the weeks of the

339 study. Again, we did not observe any differences with respect to the total amount of health

340 insurance claims $(\mathrm{p}=0.17)$, office visits $(\mathrm{p}=0.34)$, or inpatient stays $(\mathrm{p}=0.34)$. Though there was

341 slight trend towards an increase in emergency room visits among these participants in the

342 monitoring group (mean increase $=0.10$ ) compared to the controls (mean decrease $=0.12$;

$343 \mathrm{p}=0.027)$.

344

345 Health self-management

346 Additionally, we examined the relationship between monitoring/control group assignment and

347 health self-management using baseline and follow-up survey responses. We quantified

348 differences in measures of health locus of control, self-efficacy, and patient activation (Table 3).

349 Each of these are validated measures designed to address how an individual perceives his or her

350 health and health management. We did not find differences in changes in self-efficacy $(p=0.85)$ 
351 or patient activation ( $\mathrm{p}=0.68)$ between groups. In both cases, both the control and monitoring

352 groups did not differ between baseline and follow-up. The average Stanford Patient Education

353 Research Center (PERC) 6-item self-efficacy scale was 7.9 and 8.0 across both groups at

354 baseline and follow-up, respectively. Meanwhile, the average Patient Activation Measure 13-

355 item measure was 73 and 76 across both groups at baseline and follow-up, respectively.

356 However, one component of Form C of the Multidimensional Health Locus of Control (MHLC)

357 18-item scale, the propensity to view health status as due to chance factors (MHLC Chance),

358 showed improvement in the intervention group as compared to controls $(\Delta=2.06 ; \mathrm{p}=0.020)$. We

359 simultaneously observed an approximately 1.3 increase in the scale in the control arm and 0.8

360 decrease in the intervention arm. Thus, compared to controls, participants in the intervention arm

361 were less likely to view their health status as due to chance. We did not observe any group

362 differences with respect to the other health locus of control components. In each group, the

363 average scores at the follow-up visit were within 0.5 of the baseline scores (Table 3). 
Table 3. Mean values of health self-management outcomes of study. Standard deviation in parentheses. MHLC $=$ Multidimensional

Health Locus of Control; PERC $=$ Patient Education Research Center.

\begin{tabular}{|c|c|c|c|c|c|c|c|c|}
\hline & \multicolumn{2}{|l|}{ Baseline } & \multicolumn{2}{|l|}{ Follow-up } & \multicolumn{2}{|c|}{ Mean Difference } & \multirow{2}{*}{$\begin{array}{l}\text { Effect } \\
\text { Size }\end{array}$} & \multirow[b]{2}{*}{$\mathbf{p}$} \\
\hline & $\begin{array}{l}\text { Control } \\
N=85\end{array}$ & $\begin{array}{l}\text { Monitoring } \\
N=75\end{array}$ & $\begin{array}{l}\text { Control } \\
N=65\end{array}$ & $\begin{array}{l}\text { Monitoring } \\
N=65\end{array}$ & $\begin{array}{l}\text { Control } \\
N=65\end{array}$ & $\begin{array}{l}\text { Monitoring } \\
N=65\end{array}$ & & \\
\hline MHLC Internal & $26.0(6.0)$ & $26.1(6.7)$ & $26.3(6.0)$ & $26.1(5.9)$ & $0.08(6.4)$ & $0.34(5.3)$ & 0.11 & 0.80 \\
\hline MHLC Chance & $12.3(5.9)$ & $12.3(5.6)$ & $13.4(5.8)$ & $11.3(5.3)$ & $1.30(5.0)$ & $-0.76(4.9)$ & -0.93 & 0.02 \\
\hline MHLC Doctor & $14.9(2.7)$ & $15.3(2.6)$ & $14.8(3.0)$ & $15.7(2.3)$ & $-0.22(3.8)$ & $0.43(2.5)$ & 0.37 & 0.34 \\
\hline MHLC Others & $8.4(3.6)$ & $7.6(3.0)$ & $8.1(3.3)$ & $7.9(3.1)$ & $-0.15(3.8)$ & $0.50(3.2)$ & 0.35 & 0.59 \\
\hline PERC Self-Efficacy & $7.5(2.0)$ & $8.4(1.4)$ & $7.8(1.7)$ & $8.4(1.7)$ & $0.31(2.1)$ & $-0.05(1.4)$ & -0.27 & 0.85 \\
\hline Patient Activation & $70.2(14.2)$ & $77.6(13.1)$ & $74.6(18.9)$ & $79.0(20.9)$ & $4.35(18.2)$ & $0.75(18.4)$ & -0.84 & 0.68 \\
\hline
\end{tabular}


368 Among the 138 individuals who enrolled in the study with a prior indication of hypertension, we

369 obtained both baseline and follow-up systolic and diastolic blood pressure readings on 112

370 participants ( $n=61$ monitoring; $n=61$ control). The average systolic blood pressure did not differ

371 between baseline and end-of-study in the monitoring group $(\mathrm{p}=0.32)$, control group $(\mathrm{p}=0.12)$, or

372 between groups $(\mathrm{p}=0.56)$. However, the average diastolic blood pressure dropped $3.6 \mathrm{mmHg}$ in

373 the monitoring group $(\mathrm{p}=0.035)$ and $6.1 \mathrm{mmHg}$ in the control group $(\mathrm{p}=0.0036)$; though again

374 there was no difference between groups $(p=0.35)$. Likewise, among the 47 individuals who

375 enrolled in the study with a prior indication of diabetes, we obtained hemoglobin A1c levels on

37631 participants at both time points. However, hemoglobin A1c levels did not differ between

377 baseline and the end-of-study within each group or between groups $(p=0.98)$.

378

379

Device usage

380 Study participants in the monitoring group who completed the follow-up study visit used one of 381 the monitoring devices a total of 10,305 times (Figure S9). This includes 6,356 blood pressure 382 readings, 3,440 blood glucose readings, and 509 arrhythmia readings. The average number of

383 blood pressure readings was $151(\mathrm{sd}=84$; median=154) with a maximum of 436 . Four of 42

$384(10 \%)$ study participants had fewer than three times measurements over the course of the study.

385 All others had more than 60 measurements. The average number of blood glucose readings was

$386248(\mathrm{sd}=268$; median=125). Four of $14(29 \%)$ study participants did not record a reading.

387 Meanwhile, the average number of arrhythmia readings was $57(\mathrm{sd}=54$; median=53) with one 388 individual of nine (11\%) not using the device.

390 DISCUSSION 
391 Our study constitutes a major advancement over existing studies that have examined mobile

392 health technologies by virtue of its design features. First we deployed a gold-standard

393 prospective, randomized design with an intervention that included multiple key components

394 relevant for management of three chronic conditions with high morbidity and mortality. This

395 intervention included the use of three state-of-the-field wireless smartphone-enabled remote

396 monitoring medical devices. Furthermore, data from the devices was aggregated using the

397 Qualcomm Life cloud-to-cloud data integration capability. Data visualization was then provided

398 to study participants through an online care coordination application where participants could

399 view their device readings through web and mobile mediums throughout the course of the

400 intervention period. Thus, we feel that compared to previous studies in the mobile health space,

401 our intervention more closely mirrors a future in which chronic disease monitoring using mobile

402 biomedical sensors is embraced by the health care community. This requires a system that brings

403 together device manufacturers, mobile health telecommunication expertise, health care providers,

404 and employee wellness programs - all of which we utilized in the development and

405 implementation our mobile health monitoring intervention.

406

407 We enrolled 160 study participants in the study, achieving low drop-out particularly in the

408 monitoring group where $87 \%$ of participants completed all aspects of the study. We also had

409 relatively good compliance among individuals in the monitoring group. Hypertensive study

410 participants on average recorded one blood pressure measurement per day, roughly what we

411 requested (twice per day, three days per week; 6 total per week). In total, individuals in the

412 monitoring group provided over 9,000 blood pressure, blood glucose, and electrocardiogram

413 readings which we will are now examining for interesting trends in the entirety of data we 
414 collected. Interestingly, these individuals used the HealthyCircles online disease management

415 program somewhat sparingly (3,670 uses over six months for 65 study participants). Instead,

416 many users preferred the mobile tracking applications. Future research could explore different

417 means of providing data back to individuals monitoring their condition.

418

419 Overall we found little in terms of differences in health insurance claims between individuals

420 enrolled in the control and monitoring arm. This is significant because we were powered to

421 detect a moderate difference - approximately a doubling of health insurance claims dollars. This

422 suggests that while there may be small short-term increases in health care utilization as a result

423 of mobile health monitoring, there is likely not a major effect. Our equivalence testing results

424 reiterated this finding. We also expect that any short-term effect would decrease over time as a

425 user's comfort with monitoring and understanding of their data improves. Importantly, our six

426 month study period fails to capture the potential competing long-term decrease in health care

427 utilization that may occur as a result of monitoring leading to improved health management and

428 health outcomes. Taken collectively, we feel any apprehension directed at consumer mobile

429 health monitoring with respect to over-utilization of health care resources should be tempered.

430

431 Meanwhile, we found some evidence of improved health self-management in individuals who

432 received the intervention, which was characterized by a decrease in the propensity to view health

433 status as due to chance factors. One possible explanation is that this shift was due to the ability to

434 remotely, and at will, track personal biometric indices important for one's condition. Another

435 explanation is that the actual information gleaned from the readings prompted the users to

436 consider how they might make behavioral changes that would impact those metrics. Clarifying 
437 this mechanism of action could enable the development of future digital medicine interventions

438 that are refined in such a way as to optimally impact health locus of control.

440 We encountered several challenges executing this project as a result of its complexity. One 441 particular challenge was effectively dealing with the involvement of and collaboration between

442 multiple entities, including industry, research, and clinical partners in the digital medicine space.

443 Necessary legal agreements, data pipelines, and working arrangements were required to facilitate

444 the study initiation and execution. In total, the study involved over five different Scripps

445 departments, ten different companies, development and execution of at least eight different

446 contracts or legal agreements, five different terms of use that a study participant could potentially

447 have to agree to, and creation of six participant instruction or "set-up" guides. As this

448 demonstrates, the conduct and deployment of digital medicine trials can present unique

449 challenges that future work in this area could help address. We also encountered technological

450 issues. Out of 75 individuals enrolled in the monitoring group, 21 (28\%) experienced issues that

451 required the research team to log at least one help desk ticket due to technical issues with the

452 participant's phone, device(s), or connection to the online portal. Furthermore, 10 of these

453 individuals had more than one help desk ticket submitted and at least 20 individuals had to have

454 either the iPhone and/or the device replaced altogether. Technical issues are, of course, inevitable

455 when pursuing innovative interventions that leverage new technologies. However, in order for

456 such interventions to effectively provide benefit to the user they have to be seamless in order to

457 minimize participant fatigue. Exposing study participants to such issues has the potential to

458 create biases in the study results. Though we did not observed a difference in drop-out rate

459 between individuals who submitted a help desk ticket and those that did not $(\mathrm{p}=0.27)$, 
460 minimizing technological issues should be an important consideration in the design of future

461 digital medicine trials.

462

463 In conclusion, we have presented the first prospective randomized trial of a digital medicine

464 intervention with multiple smartphone-enabled biosensors, data aggregated and visualized

465 through an online connected health platform, deployed with three high morbidity and mortality

466 chronic diseases examining health care utilization. Our results suggest there is little to no short-

467 term increase in health care utilization as a result of participation in a comprehensive mobile

468 health monitoring care coordination platform. Meanwhile, we did see some improvement in

469 health self-management. Future work should explore the potential reduction in long-term health

470 care utilization as a result of potentially improved health management due to mobile health

471 monitoring.

472 
473

474

475

476

477

478

479

480

481

482

483

484

485

486

487

488

489

490

491

492

493

494

495

496

497

498

499

500

501

502

503

504

505

506

\section{REFERENCES}

American Diabetes Association. 2014. National Diabetes Statistics Report, 2014 Estimates of Diabetes and Its Burden in the Epidemiologic estimation methods. National Diabetes Statistics Report:2009-2012.

Boeldt DL., Wineinger NE., Waalen J., Gollamudi S., Grossberg A., Steinhubl SR., McCollisterSlipp A., Rogers MA., Silvers C., Topol EJ. 2015. How Consumers and Physicians View New Medical Technology: Comparative Survey. Journal of medical Internet research $17: \mathrm{e} 215$.

Chobanian A V., Bakris GL., Black HR., Cushman WC., Green L a., Izzo JL., Jones DW., Materson BJ., Oparil S., Wright JT., Roccella EJ. 2003. Seventh report of the Joint National Committee on Prevention, Detection, Evaluation, and Treatment of High Blood Pressure. Hypertension 42:1206-1252.

Davis KE. 2013.Expenditures for Hypertension among Adults Age 18 and Older, 2010: Estimates for the U.S. Civilian Noninstitutionalized Population April 2013. Available at http://meps.ahrq.gov/mepsweb/data_files/publications/st404/stat404.shtml

Ernst ME., Carter BL., Goerdt CJ., Steffensmeier JJG., Phillips BB., Zimmerman MB., Bergus GR. 2006. Comparative antihypertensive effects of hydrochlorothiazide and chlorthalidone on ambulatory and office blood pressure. Hypertension 47:352-358.

Free C., Phillips G., Galli L., Watson L., Felix L., Edwards P., Patel V., Haines A. 2013a. The Effectiveness of Mobile-Health Technology-Based Health Behaviour Change or Disease Management Interventions for Health Care Consumers: A Systematic Review. PLoS Medicine 10:e1001362.

Free C., Phillips G., Watson L., Galli L., Felix L., Edwards P., Patel V., Haines A. 2013b. The effectiveness of mobile-health technologies to improve health care service delivery processes: a systematic review and meta-analysis. PLoS medicine 10:e1001363.

Guyatt G., Sackett D., Taylor DW., Chong J., Roberts R., Pugsley S. 1986. Determining optimal therapy--randomized trials in individual patients. The New England journal of medicine 314:889-892.

Hamine S., Gerth-Guyette E., Faulx D., Green BB., Ginsburg AS. 2015. Impact of mHealth Chronic Disease Management on Treatment Adherence and Patient Outcomes: A Systematic Review. Journal of Medical Internet Research 17:e52.

Hansen TW., Jeppesen J., Rasmussen S., Ibsen H., Torp-Pedersen C. 2005. Ambulatory blood pressure and mortality: A population-based study. Hypertension 45:499-504.

Hibbard JH., Mahoney ER., Stockard J., Tusler M. 2005. Development and testing of a short 
form of the patient activation measure. Health Services Research 40:1918-1930.

508 Karhula T., Vuorinen A-L., Rääpysjärvi K., Pakanen M., Itkonen P., Tepponen M., Junno U-M.,

509

510

512

513

514

515

516

517

518

519

520

521

522

523

524

525

526

527

528

529

530

531

532

533

534

535

536

537

538

539

540 Jokinen T., van Gils M., Lähteenmäki J., Kohtamäki K., Saranummi N. 2015. Telemonitoring and Mobile Phone-Based Health Coaching Among Finnish Diabetic and Heart Disease Patients: Randomized Controlled Trial. Journal of medical Internet research 17:e153.

Kim MH., Johnston SS., Chu B-C., Dalal MR., Schulman KL. 2011. Estimation of total incremental health care costs in patients with atrial fibrillation in the United States. Circulation. Cardiovascular quality and outcomes 4:313-320.

Lorig K., Chastain RL., Ung E., Shoor S., Holman HR. 1989. Development and evaluation of a scale to measure perceived self-efficacy in people with arthritis. Arthritis and rheumatism 32:37-44.

National Institute for Health and Care Excellence 2015. Available at https://www.nice.org.uk/ (accessed October 12, 2015).

Sander GE., Giles TD. 2011. Resistant hypertension: Concepts and approach to management. Current Hypertension Reports 13:347-355.

Steinhubl SR., Muse ED., Topol EJ. 2013. Can mobile health technologies transform health care? JAMA : the journal of the American Medical Association 310:2395-6.

Steinhubl SR., Muse ED., Topol EJ. 2015. The emerging field of mobile health. Science translational medicine 7:283rv3.

The ALLHAT Officers. 2002. Major Outcomes in High-Risk Hypertensive Patients Randomized to or Calcium Channel Blocker vs Diuretic. Journal of the American Medical Association 288:2981-2997.

The ALLHAT Officers and Coordinators for the ALLHAT Collaborative Research Group. 2000. Major Cardiovascular Events in Hypertensive Patients Randomized to Doxazosin vs Chlorthalidone. JAMA: The Journal of the American Medical Association 283:1967-1975.

Uzu T., Harada T., Namba T., Yamamoto R., Takahara K., Yamauchi A., Kimura G. 2005. Thiazide diuretics enhance nocturnal blood pressure fall and reduce proteinuria in immunoglobulin A nephropathy treated with angiotensin II modulators.

Wallston KA., Stein MJ., Smith CA. 1994. Form C of the MHLC scales: a condition-specific measure of locus of control. Journal of personality assessment 63:534-553.

Willits I., Keltie K., Craig J., Sims A. 2014. WatchBP Home A for opportunistically detecting atrial fibrillation during diagnosis and monitoring of hypertension: A NICE medical technology guidance. Applied Health Economics and Health Policy 12:255-265. 
541 Wilson PWF., D’Agostino RB., Levy D., Belanger AM., Silbershatz H., Kannel WB. 1998.

542 Prediction of Coronary Heart Disease Using Risk Factor Categories. Circulation 97:1837-

$543 \quad 1847$.

544 Yang W., Dall TM., Halder P., Gallo P., Kowal SL., Hogan PF., Petersen M. 2013. Economic 545 costs of diabetes in the U.S. in 2012. Diabetes Care 36:1033-1046.

546 Zhang B., Kumar RB., Dai H., Feldman BJ. 2014. A plasmonic chip for biomarker discovery and 547 diagnosis of type 1 diabetes. Nature Medicine 20:948-953. 